\title{
Heisenberg’s Versus Von Neumann’s Indeterminism of Quantum Mechanics
}

\author{
Franz Klaus Jansen \\ Independent Researcher
}

Irreducible indeterminism is considered by most physicists as an ontological interpretation of quantum mechanics, which attributes inherent indeterminism to elementary particles. This view was extrapolated by von Neumann from the atomocosm to the entire universe. Heisenberg proposed an epistemic interpretation, postulating that indeterminism stems from ambivalent detection systems, rather than being a characteristic of elementary particles. Transformative detection that inherently affects the measured phenomena is responsible for the indeterminism in the atomocosm. As the same does not generally hold true in the macrocosm, this discrepancy has led to the notion of the Heisenberg cut. As explained by Heisenberg's microscope example, high-energy light can displace electrons from their atomic shells in the atomocosm, but would not induce the same effect on objects in the macrocosm, thus rendering such detection neutral. Therefore, detection systems are ambivalent in that they can be transformative under the cut and neutral otherwise. Device variation is found under and above the cut and is the essential cause of outcome variability in the macrocosm. Thus, two completely different categories of indeterminism exist simultaneously under the Heisenberg cut, but only one is found above the cut, known as measurement variations of devices. Experimental exploration of elementary particle behaviors is possible only with the help of detection systems. If these systems affect particle characteristics in any way, this would be sufficient to explain the irreducible quantum mechanical indeterminism. Consequently, the true behavior of elementary particles, whether indeterminist or determinist, would never be detectable. Above the cut, on the other hand, variations in device performance are inevitable, due to reducible perturbing factors, inducing measurement variation of devices. Heisenberg discovered a general principle of ambivalent detection systems, which can also be found in the macrocosm. In the Wilson Cloud Chamber, vapor is an ambivalent detection system, since high-energy charged particles would produce straight tracks, whereas those of lower energy would leave an irregular trace. According to the epistemic interpretation, von Neumann's extrapolation of irreducible quantum mechanical indeterminism to the entire universe would not be necessary, thereby avoiding the uncomfortable conclusion that the entire universe is based on indeterminism.

Keywords: quantum mechanics, indeterminism, ontic interpretation, extrapolation to universe, epistemic interpretation, Heisenberg cut, detection systems, device variations

\section{Introduction}

Heisenberg (1927) described the uncertainty principle, postulating that, at the scale of elementary particles,

Franz Klaus Jansen, MD, Ph.D., former: Biological Research in Immunology at the University of Düsseldorf, Germany, and later SANOFI, Pharmaceutical Company in Montpellier, France; now main research field: Philosophy of Science. 
location and velocity cannot simultaneously be determined with precision. Schrödinger's (1926) wave function is a mathematical tool for transforming quantum mechanical indeterminacy with a formalism based on linear superposition in a Hilbert space into probability estimations. According to Heisenberg and the Copenhagen interpretation, superposition in the wave function is reduced by the measurement, producing one measurable outcome (Heisenberg 1927). The wave function reduction was differently interpreted by several physicists. Ghirardi, Rimini, and Weber (1985) and Penrose (1994) described it as spontaneous objective collapse. Zurek (1981) proposed decoherence of the wave function by interaction with the environment, while a hidden variable theory was introduced by Bohm (1952). Some interpretations do not require any collapse, so that the wave function exists in multiple parallel entities as multiple worlds or multiple minds (Everett 1957; Zeh 1970). More recently, following Everett's interpretation, Tegmark (2014) considered the universe at different levels, the last of which consists of pure mathematics. Dorato $(2015,3)$ interpreted the wave function as an abstract mathematical entity distinct from physical entities existing in spacetime, asserting that "whatever is denoted by the wave function exists abstractly and not in spacetime.”

Von Neumann (1955) extrapolated the wave function and thereby quantum mechanical indeterminism to the entire universe. In this perspective, quantum mechanical indeterminism is interpreted as an inherent property of elementary particles, which is an ontic interpretation of quantum mechanics. Leading physicists have generally accepted the ontic view, proposing more than 15 different interpretations (Cabello 2016).

Besides its application in physics, quantum mechanical formalism has also been successfully employed in other disciplines when treating uncertainties, such as human decision making (Aerts, D’Hooghe, \& Sozzo 2011; Busemeyer \& Bruzza 2011; Pothos \& Busemeyer 2009). Thus, kind of isomorphism exists between some interpretations of quantum mechanics and consciousness (Jansen 2008), as well as between the uncertainty observed in quantum mechanics and the one encountered when making predictions in everyday life (Jansen 2011).

Heisenberg (1927) explained epistemic indeterminism using a theoretical microscope as an example, whereby high-energy light directly interacts with elementary particles and modifies their location and velocity. In this thought experiment, light becomes a transformative detection for elementary particles. In addition, the variation inherent in imperfect devices contributes to observable indeterminism. Heisenberg separated a lower-level from a higher-level scale of observable indeterminism, thus introducing the notion of Heisenberg cut, separating the phenomena subject to transformative detection and device variation from those that are subject to device variation only. Heisenberg's (1927) epistemic interpretation of quantum mechanics is limited to the methods by which our knowledge on elementary particles is acquired. Thereby, the cause of indeterminism is shifted from elementary particles to their detection systems, but only within a limited scale. This approach avoids the extrapolation of quantum mechanical indeterminism to the entire universe, as proposed by von Neumann (1955). Inadequacy between physical objects and their detection systems is not only a quantum mechanical problem, but a general problem, since it is also found in the macrocosm.

\section{Regularity and Irregularity of Physical Observations}

Physical exploration is based on consistent and congruent scientific observations. Scientific laws signify that the same interactions between physical factors can be observed repeatedly, whereby this consistency in findings forms the basis of scientific laws. Nevertheless, when experiments are repeated under exactly the same conditions, they rarely lead to completely identical results, due to a wide range of perturbing factors that are 
beyond the scientists' control. This discrepancy in outcomes can be attributed to the high complexity of physical as well as biological experiments, where multiple influential factors cannot be avoided.

\section{Quantum mechanical indeterminism}

I.

ontological :
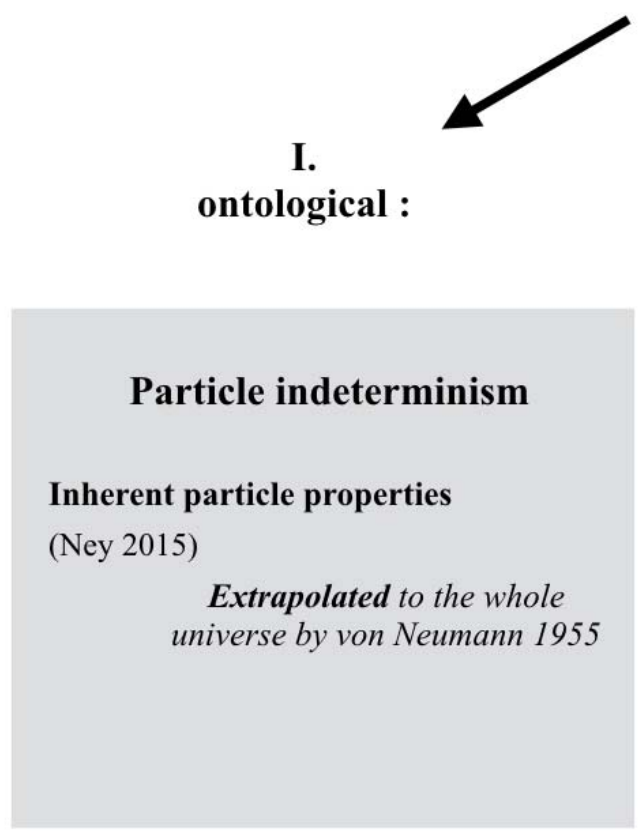

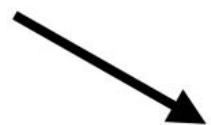

II.

epistemic :

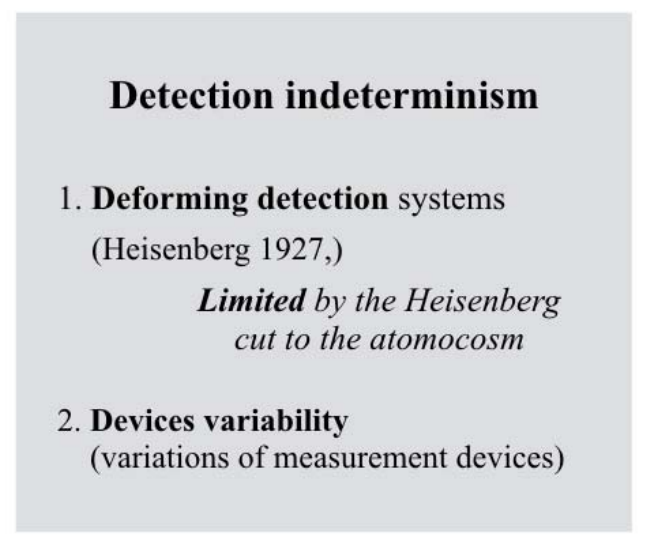

Fig. 1. Interpretations of quantum mechanical indeterminism.

There is a distinction between ontological and epistemic quantum mechanical indeterminism. Ontological quantum mechanical indeterminism is an inherent particle property, which is irreducible and can be extrapolated to the entire universe. Epistemic indeterminism is no longer a particle property; rather, it is produced by transformative detection that modifies the behavior of particles being examined. It is also irreducible, but is limited to the area under the Heisenberg cut and cannot be extrapolated to the entire universe.

In scientific experiments, the aim is to establish the link between input and output parameters. Nevertheless, multiple confounding factors that are not accounted for by the experimental design are simultaneously present. Therefore, conclusive results can only be obtained if these factors are eliminated or at least remain constant during the experimentation process. Since this is rarely possible, identical results are never obtained in repeated experiments. In classical physics, variations are generally small and are classified as manipulation errors, such as in Galilee's experiments on the inclined plane (Straulino 2008). Since mathematics is exempt of even the slightest variations during repetition, it is deemed to better represent the expected true behavior of nature. Therefore, Galilee proposed replacing small variations in manipulation errors implicit in experimental results by mathematical concepts. Correspondingly, Galilei (1623, 237-238) wrote, "This book [the universe] is written in the mathematical language, and the symbols are triangles, circles, and other geometrical figures.”

Repetitions under identical experimental conditions resulting in unavoidable (smaller or greater) variations in outcomes hinder unambiguous interpretation. Nevertheless, device variations can be reduced by designing more reliable devices, although complete elimination of variations is never possible in complex physical or 
biological experiments. Although in the simple experiments conducted by Galilee, small manipulation errors could not be avoided, even today, when experiments are much more complex, highly sophisticated devices will inevitably contribute to variations in outcomes. In classical physics, perturbing factors induce random variations, causing uncertainty in the results.

However, quantum mechanical indeterminism is interpreted differently. According to the ontological interpretation, the particles are non-deterministic. For Albert $(2013,53)$, quantum mechanics are concrete physical objects and for Ney $(2016,2)$, the wave function is “a field in a high dimensional space," as summarized in Figure 1.

Heisenberg's contrasting epistemic interpretation of quantum mechanics posited that our knowledge of elementary particles is dependent on transformative detection. Consequently, even if elementary particles are deterministic by nature, inadequate detection systems will result in non-deterministic outcomes. Heisenberg's microscope thought experiment offers an epistemic interpretation of quantum mechanics, which could be composed of two types—reducible and irreducible—of indeterminism. The reducible part corresponds to classical indeterminism, induced by perturbing factors resulting in the variations implicit in the measurement devices, which can theoretically be diminished by designing better devices. The irreducible part arises due to modifications that detection systems induce in experimental outcomes in the atomocosm. Nevertheless, they are limited to the area below a boundary, called the Heisenberg cut, and cannot be extrapolated to the entire universe.

\section{Neutral and Transformative Detection}

Experiments are repeated to ascertain that the same results can be consistently obtained. In classical physics experiments, such as Galilee's experiment on the inclined plane (Straulino 2008), the potential variations or manipulation errors are relatively small. In quantum mechanical experiments, variations obtained under identical experimental conditions are considerable and can be interpreted as ontological or as epistemic indeterminism.

Heisenberg was of view that, at the level of elementary particles, observation by physical methods directly interferes with the explored particles, thereby changing the observable outcomes, which leads to uncertainty. Heisenberg (1933, 292) illustrated this principle by a thought experiment with a hypothetical microscope, capable of detecting electrons. He stated, "the first light quantum from the light source to reach the electron and pass into the observer's eye would eject the electron completely from its path.” He also noted (297), "The perturbation of the system to be observed caused by the observation is also an important factor in determining the limits within which a visual description of atomic phenomena is possible.”

Whereas ontological interpretations consider the observed indeterminism as an inherent property of elementary particles in the atomocosm, which can be extrapolated to the entire universe, Heisenberg's epistemic interpretation limits quantum mechanical indeterminism to ambivalent detection systems capable of interfering with the observed phenomena under the Heisenberg cut (Figure 2). Nevertheless, ontological and epistemic indeterminism can be mathematically treated using the same formalism, Schrödinger's (1926) wave function, so that their distinction becomes purely philosophical. The potential cause of indeterminism could therefore be considered as an inherent property of elementary particles, or solely as the consequence of employing transformative detection. 


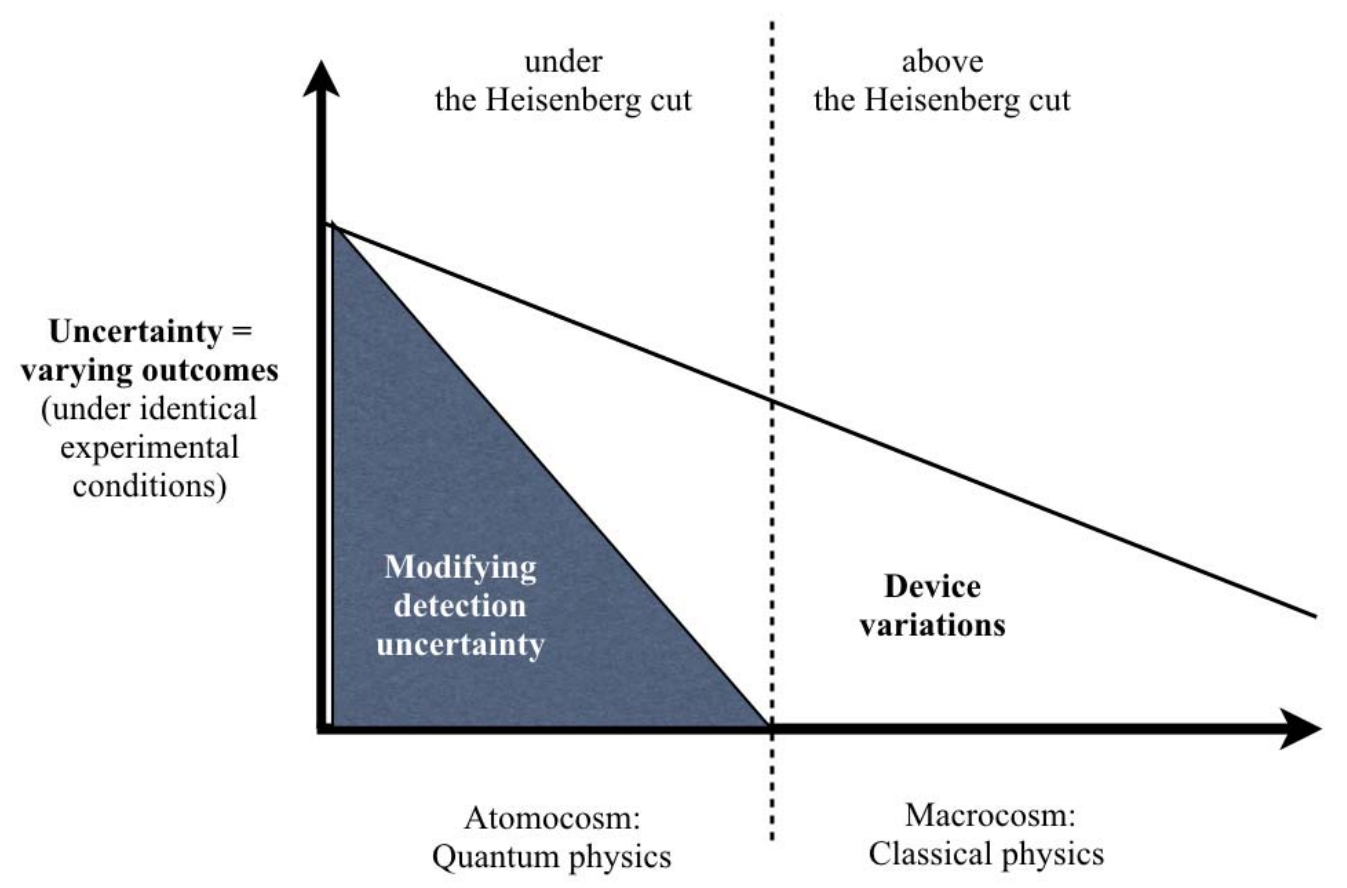

Fig. 2. The Heisenberg cut.

The Heisenberg cut limits indeterminism of quantum mechanics to transformative detection plus device variations under the cut in the atomocosm. Above the cut, there is essentially device variation, as found in classical physics and biology.

In recent years, Heisenberg's uncertainty equation has been criticized. Ozawa (2003) proposed an alternative equation, better adapted for the estimation of the measurement disturbance relation under certain experimental conditions. Lund and Wiseman (2010), as well as Rozema et al. (2013), demonstrated experimentally that the threshold of Heisenberg's uncertainty could be lower than initially proposed. Thus, Heisenberg's uncertainty principle is maintained, albeit under a lower threshold.

If a detection system is transformative for measurement of the properties of target factors, it induces Heisenberg's uncertainty. In the macrocosm of classical physics, detection is in general neutral and does not perturb the measurement procedure during an experiment, thus permitting an undisturbed transmission of information. Heisenberg's thought experiment pertaining to the atomocosm implies that high-energy light may affect electron properties, although light as the detection system in the macrocosm cannot move objects. Therefore, detection systems such as light are ambivalent, whereby they provide neutral detection in the macrocosm, while affecting particle properties in the atomocosm under the Heisenberg cut.

\section{Neutral and Transformative Detection in the Macrocosm}

Although Heisenberg's epistemic interpretation suggests that exploration of target properties under the Heisenberg cut is inevitably affected by ambivalent detection systems, a similar disturbance can also be found above the cut in the macrocosm, for instance when elementary particles interact with macroscopic vapor (Figure 3). In the Wilson Cloud Chamber (Wikipedia, Cloud Chamber) containing supersaturated water or alcohol vapor, alpha particles, beta rays, muons, protons and electrons cause ionization of gas particles, which 
act as condensation nuclei, inducing water droplets. The vapor in the chamber represents a macroscopic detection system for highly energetic charged elementary particles. Alpha particles, high-energy muons and protons induce a straight line of droplets, but low-energy electrons produce thin and irregularly shaped traces. The vapor in the Wilson Cloud Chamber represents a neutral detection system for high-energy charged particles that produce straight tracks, but becomes a transformative detection system for low-energy electrons inducing irregular tracks. This is an example of an ambivalent macroscopic detection system.

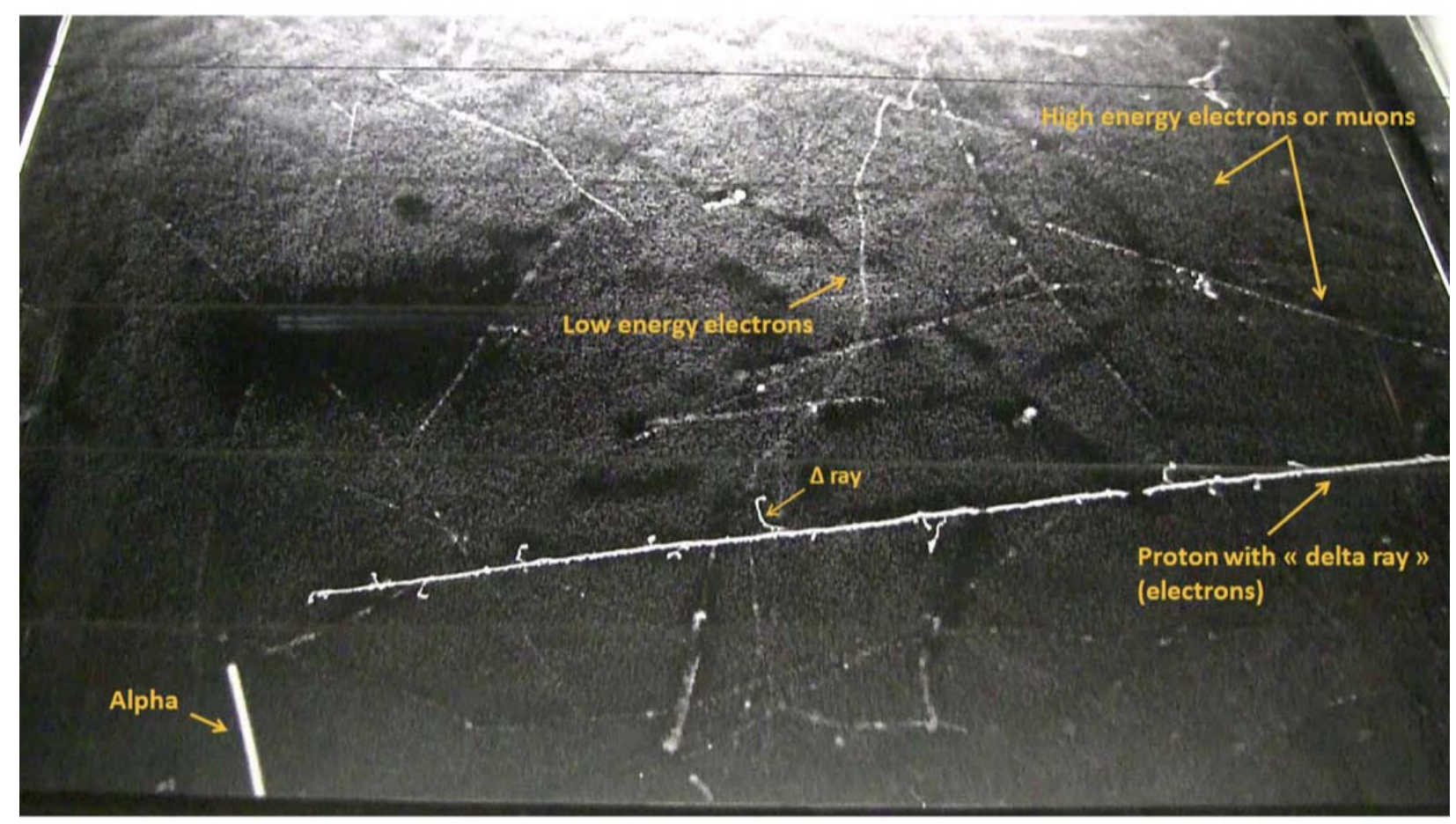

Fig. 3. Wilson Cloud Chamber with four types of charged elementary particles.

Wikipedia: Cloud Chamber, picture taken at the Pic du Midi, showing electrons, muons, protons and alpha particles with an ambivalent detection system. Muons, protons and alpha particles induce strait tracks formed by droplets with neutral detection, whereas low energy electrons create irregular traces by transformative detection.

Under some rare conditions, transformative detection also leads to uncertainties in the macrocosm. When a blind man tries to localize a football on the ground with a blind crutch, the crutch will displace the football when it comes in contact with it, but would not have the same effect on much heavier objects (Figure 4). 


\begin{tabular}{|c|c|c|}
\hline Examples & $\begin{array}{c}\text { Heisenberg Cut } \\
\text { in } \\
\text { Atomocosm }\end{array}$ & $\begin{array}{c}\text { Generalized cut } \\
\text { in } \\
\text { Macrocosm }\end{array}$ \\
\hline $\begin{array}{c}\text { Heisenberg's } \\
\text { overpowered } \\
\text { microscope }\end{array}$ & $\begin{array}{c}\text { electrons } \\
\text { mass level of targets } \\
\text { (electron / football) }\end{array}$ & \\
\hline $\begin{array}{c}\text { Wilson chamber } \\
\text { Blind man }\end{array}$ & retion by light \\
& & $\begin{array}{c}\text { radiation particles } \\
\text { energy level of targets } \\
\text { (protons / electrons) }\end{array}$ \\
\hline
\end{tabular}

Fig. 4. Generalization of Heisenberg's “transformative detection.”

Transformative detection can be found in the atomocosm as well as in the macrocosm, for instance in the Wilson Cloud Chamber or with the blind crutch. It depends on the relation of an ambivalent detection system to its targets, if they have significantly different mass levels, energy levels or mobility levels.

\section{Extrapolation of Indeterminism to the Entire Universe}

Von Neumann $(1955,17)$ extrapolated quantum mechanical indeterminism to the entire universe. Schwartz, Stapp, and Beauregard wrote:

All three of the alternative approaches [referring to those put forth by Penrose, Everett, Bohm] accept von Neumann's move of treating the entire physical world quantum mechanically. In particular, the bodies and brains of the agents are treated as conglomerations of such things as quantum mechanically described electrons, ions and photons. $(2005,1325)$

This interpretation corresponds to an unlimited extrapolation of the mathematical formalism of the wave function, which is considered to represent reality. The interpretation of inherent indeterminism of elementary particles corresponds to this ontological interpretation of the wave function.

Von Neumann's ontic interpretation of the wave function did not differentiate among particle properties, transformative detection, and device variations, but considered quantum mechanical indeterminism only as an inherent property of elementary particles. Since the universe consists of elementary particles, it should also depend on quantum mechanical indeterminism. However, Heisenberg's epistemic interpretation only allows transformative detection by light under the cut, where light quanta could displace elementary particles, but not above the cut for the observation of objects in the macrocosm. Therefore, epistemic interpretation does not 
require extrapolation of quantum mechanical indeterminism to the macrocosm, and eliminates the weird aspect of the ontological interpretation that the entire universe is inherently non-deterministic.

The ontological interpretation of inherent indeterminism of elementary particles is indeed tempting, since it could easily explain the observed indeterminate outcomes in quantum mechanical experimentation. However, observation and interpretation do not always coincide, as seen in the ancient dispute between those supporting geocentrism and the advocates for heliocentrism. The daily observation of the Sun raising in the east and setting in the west naturally suggests the interpretation that the Sun revolves around the Earth. The opposite interpretation of the Earth rotating around its axis remains unobservable, although it proved to be correct. In quantum mechanics, there is a similar situation with two possible interpretations for the same observation.

Most leading physicists accept the ontological interpretation of irreducible quantum mechanical indeterminism, suggesting an inherent indeterminism of elementary particles. In contrast, if irreducible indeterminacy is explained by an epistemic interpretation following Heisenberg's microscope thought experiment, inadequacy of the detection systems due to their interaction with targets of observation could also explain the uncertainty principle. Yet, it no longer requires the indeterminist behavior of elementary particles. Interaction of detection systems with elementary particles could explain the major part of quantum mechanical uncertainty under the Heisenberg cut and is irreducible. The remaining part is due to device variations, which can be reduced by improving devices, and is found under and above the Heisenberg cut. Consequently, with an epistemic quantum mechanical interpretation indeterminism becomes essentially the consequence of transformative detection.

The Heisenberg cut limits indeterminism to the part of the scale below the cut, where both transformative detection and device variation occur. The part of the scale above the cut pertains to neutral detection, where indeterminism is essentially due to device variability (Figure 2). By adopting an ambivalent detection system, such as light, different outcomes are produced in the atomocosm relative to the macrocosm. Specifically, under the cut, light is a transformative detection system and becomes a neutral detection system above the cut, which shows a scale dependence of the behavior of an ambivalent detection system.

Ontological or epistemic indeterminacy are only two philosophical interpretations of the same physical observations and can both be treated by Schrödinger's wave function for predicting probable experimental outcomes, and thereby confirm the high practical value of quantum mechanical formalisms. Nevertheless, the two interpretations lead to different conceptions of quantum mechanics, whereby one is extrapolated to the entire universe, while the other has a boundary at the Heisenberg cut (Figure 5).

Von Neumann's extrapolation of ontological indeterminism is at the core of some conceptions, such as spontaneous wave function collapse (Ghirardi et al. 1985), decoherence (Zurek 1981), pilot waves (Bohm 1952), parallel universes (Everett 1957), or parallel minds (Zeh 1970). In contrast, the epistemic interpretation of Heisenberg's uncertainty would avoid the extrapolation of quantum mechanical indeterminism, since it is limited to the part of the scale below the Heisenberg cut, and becomes simply insufficient knowledge of the behavior of the elementary particles themselves. 


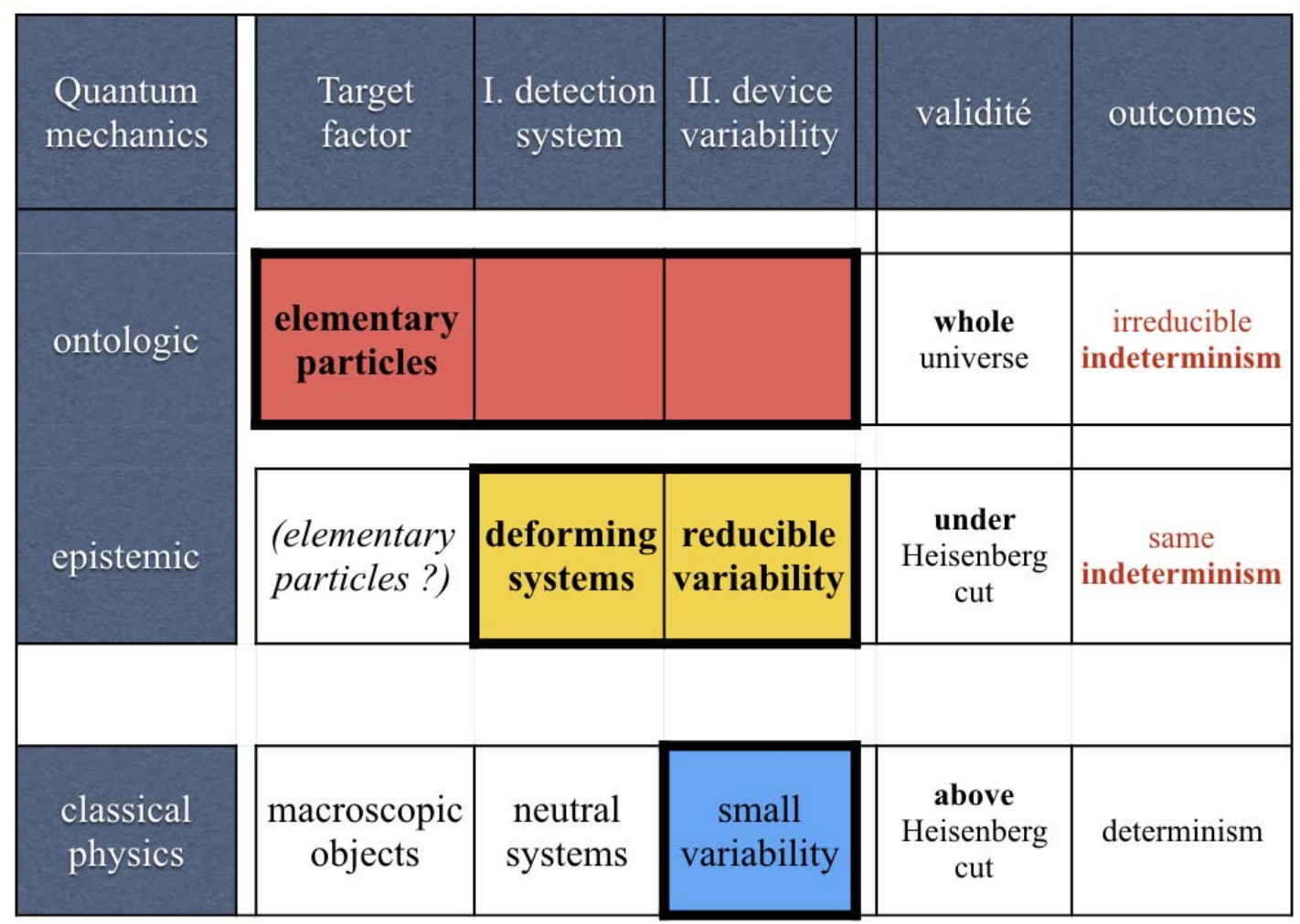

Fig. 5. Comparison of different interpretations.

Ontological and epistemic quantum mechanical interpretations are compared to each other concerning the causes of indeterminism, whereas classical physics is considered as deterministic.

In sum, the ontological interpretation only takes into account elementary particles, whereas the epistemic interpretation only considers transformative detection, which permits no access to the particle properties (Figure 5). In determinist classical physics, only devices induce variability. Ontological interpretations require indeterminism for the entire universe, whereas epistemic interpretations avoid the assumption that the entire universe is indeterminist.

\section{Conclusions}

From a psycho-biological perspective, the observer plays an active role in the observation, since he/she observes the physical environment with all sensory organs and then constructs physical laws according to his/her mental representation of extra-mental reality (Jansen 2015; 2016). If perturbing factors could be eliminated or rendered immutable, multiple experiments under identical conditions would yield identical outcomes and the interaction of the object of observation with the outcome would reflect a determinist cause-and-effect relationship. However, in complex experiments, it is impossible to achieve constancy of all possible influential factors, which leads to non-deterministic outcomes. In the macrocosm, perturbing factors generally arise due to imperfections in the measurement devices, whereas in the atomocosm, the essential perturbing factors are unknown, and irreducible quantum mechanical indeterminism requires other explanations. 
Von Neumann (1955), Stapp (2001) and others considered quantum mechanical indeterminism as an inherent property of elementary particles themselves, which is an ontological interpretation. The quantum mechanical indeterminism thus had to be extrapolated to the entire universe, since it is composed of elementary particles. The wave function strengthens the extrapolation requirement, since it has no limit and is considered to reflect physical reality. Nevertheless, physical formalism sometimes overrules physical reality, which has to be verified (Sorli 2018).

Most leading physicists adopt a bottom-up view of the universe, starting with its basic constituents, the elementary particles. The final aim is to develop a "theory of everything” allowing a reconstruction of the entire universe from elementary particles with mathematical formalism, as put forth by Tegmark (2014). However, extrapolation of quantum mechanical indeterminism to the whole universe necessitates that determinism in the macrocosm be replaced by indeterminism.

Heisenberg described a different kind of indeterminism induced by transformative detection, which interferes with the detection of target properties, thereby rendering the experimental outcomes non-deterministic. Such detection systems are, however, limited to the lower part of the scale under the Heisenberg cut, where measurement variation also occurs. Transformative detection corresponds to measurement-disturbance relationship and was experimentally distinguished from the variation of the device system by Ozawa (2003), Lund and Wiseman (2010), and Rozema et al. (2012). These authors proposed that the threshold of the Heisenberg cut be set to a lower level, which Rosema et al. (2013, 3) described as, "we believe that it [Ozawa's definition] is closer in spirit to the disturbance typically associated with Heisenberg's microscope.” Thus, the Heisenberg cut was experimentally confirmed, albeit at a lower threshold.

An epistemic interpretation of quantum mechanical indeterminism, as proposed Heisenberg (1927) has two different causes, the transformative detection (I) and device variations (II) (Figure 5). Neither of these causes pertains to the inherent properties of elementary particles, as they relate solely to the way particles can be observed by a physicist. If observation methods alone create indeterminist outcomes, this would be sufficient to explain quantum mechanical indeterminism. Nevertheless, Zeh (2013, 97) wrote that “Heisenberg’s early attempts to understand his uncertainty principle simply as a consequence of unavoidable perturbations of the electron during measurements (for example by means of his electron microscope) failed as a consistent explanation.”

Irreducible transformative detection is solely attributed to atomocosm and therefore creates a boundary, called the Heisenberg cut. The physical chain of interactions starts with particle properties and interactions among particles. The chain continues with transformative detection and device variation. Consequently, only neutral detection permits accurate exploration of the particles themselves, whereas detection that modifies particle properties blocks the access to elementary particles. As a result, it can never be established if their behavior is indeterminist or determinist. In a similar sense, Plotnitsky $(2017,66)$ stated: "we cannot know or even conceive, now or possibly ever, of the ultimate character of this reality, either the reality of 'things' or the reality of the relationships between 'things' defining it."

Uncertainty caused by ambivalent detection systems represents a general principle, which Heisenberg described for the atomocosm. Under certain conditions, it can also be found in the macrocosm, such as Wilson Cloud Chamber or the blind crutch. The essential role of detection systems consists in the unmodified transmission of information from target factors through amplifying devices to sensory organs and the brain. An ambivalent detection system can vacillate between transformation and neutrality, depending on its relationship 
with the target properties. Observation with neutral detection allows the establishment of deterministic physical laws, whereas transformative detection systems only permit creation of laws with probability estimations.

A bottom-up representation of the universe from the atomocosm to the macrocosm, as physicists envisage, is based on uncertainty and is extrapolated to the entire universe (Figure 6). In contrast, a top-down representation from the level of the observer in the macrocosm to the atomocosm or a bottom-up view from the observer to the astrocosm would start with macroscopic certainty but would become increasingly uncertain. Although humans are not at the center of the universe, we do develop our knowledge on the universe from this perspective. Similar to an optical perspective, the perspective of scientific knowledge in the atomocosm and the astrocosm is subject to progressively greater uncertainty as the distance from the observer increases. Distant physical phenomena thus become mere approximations with probability estimations.

\section{Physics}

mono-directional

(from Bottom to Top)

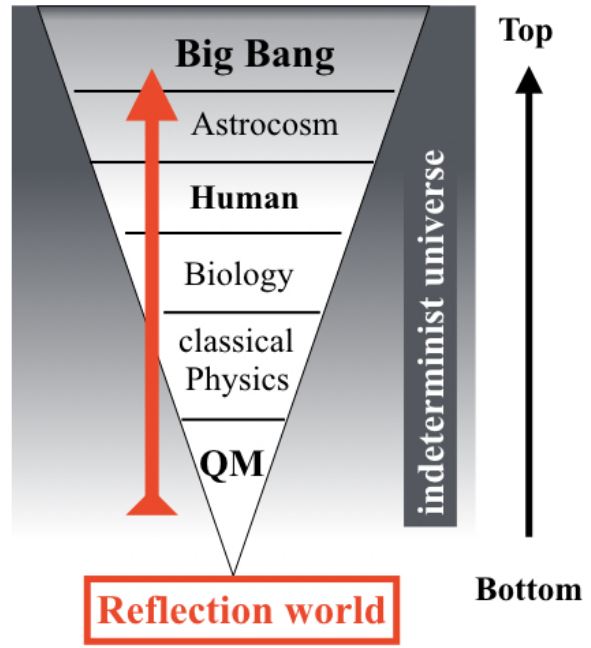

Description of nature
Bio-psychology

bi-directional :

(from the observer to Top and Bottom)

Fig. 6. Mono-directional and bi-directional worldviews.

Physics tries to construct a mono-directional reflection world according to the "theory of everything" from quantum mechanics up to the Big Bang. Therefore, the entire universe becomes indeterminist. In contrast, bio-psychology starts with the perception world of an observer in the macrocosm, which is certain, but undergoes more and more uncertainty with increasing distance from the observer in the atomocosm and the astrocosm.

\section{Works Cited}

Aerts, Diederik, D’Hooghe, Bart, \& Sozzo, Sandro. “A Quantum Cognition Analysis of the Ellsberg Paradox.” 2011. 28/06/2018. $<$ https://arxiv.org/abs/1104.1459>.

Albert, David Z. The Wave Function: Essays on the Metaphysics of Quantum Mechanics. Oxford, UK: Oxford University Press, 2013.

Bohm, David J. “A Suggested Interpretation of Quantum Theory in Terms of Hidden Variables.” Phys. Rev. 85 (1952): 166-79. 
Busemeyer, Jerome R., \& Bruza, Peter D. Quantum Models of Cognition and Decision Making. Cambridge, UK: Cambridge University Press, 2011.

Cabello, Adan. “Interpretations of Quantum Theory: A Map of Madness.” 2016. 10/1/2018. <https://arxiv.org/abs/1509.04711>.

Dorato, Mauro. "Laws of Nature and the Reality of the Wave Function.” Synthese 192.10 (2015): 3179-201.

Everett, Hugh. "Relative State Formulation of Quantum Mechanics.” Reviews of Modern Physics 29 (1957): 454-62.

Galilei, Galileo. "Il Saggiatore.” Trans. D. Stillman. The Assayer in Discoveries and Opinions of Galileo. New York, NY: Doubleday \& Company, Inc., 1623. 237-8.

Ghirardi, G. C., Rimini, A., \& Weber, T. “A Model for a Unified Quantum Description of Macroscopic and Microscopic Systems.” Eds. L. Accardi et al. Quantum Probability and Applications. Berlin, Germany: Springer, 1985. 57.

Heisenberg, Werner. "Über den anschaulichen Inhalt der quantentheoretischen Kinematik und Mechanik" (The actual content of quantum theoretical kinematics and mechanics).” Z. Phys. 43 (1927): 172-98.

---. "Nobel Lecture: The Development of Quantum Mechanics.” Nobelprize.org. 1933. 28/6/2018. $<$ http://www.nobelprize.org/nobel_prizes/physics/laureates/1932/heisenberg-lecture.html>.

Jansen, Franz K. "Partial Isomorphism of Superposition in Potentiality Systems of Consciousness and Quantum mechanics.” Neuro Quantology 6.3 (2008): 278-88.

---. “Isomorphic Concepts for Uncertainty Between Consciousness and Some Interpretations of Quantum Mechanics.” Neuro Quantology 9.4 (2011): 660-68.

---. “Quantum Mechanics Is Not Physical Reality But Mental Potentiality Because of the Law of Non-contradiction.” Neuro Quantology 13.1 (2015): 79-89.

---. “Elementary Sensation Primacy Creating a First to Third Person Gap of Consciousness.” Intern J Psychol Research 10 (2016): $1-25$.

Lund, A. P., \& Wiseman, H. W. “Measuring Measurement-Disturbance Relationships With Weak Values.” New Journal of Physics 12 (2010): 093011.

Ney, Alyssa. "Finding the World in the Wave Function.” 2016. 10/1/2018. <http://philsci-archive-dev.library.pitt.edu/11877/>.

Ozawa, Mazanao. "Universally Valid Reformulation of the Heisenberg Uncertainty Principle on Noise and Disturbance in Measurement.” Phys. Rev. A 67 (2003): 042105.

Penrose, Roger. Shadows of the Mind. New York, NY: Oxford University Press, 1994.

Plotnitsky, Arkady. “Structure Without Law: From Heisenberg’s Matrix Mechanics to Structural Nonrealism.” Mind and Matter 16.1 (2017): 59-96.

Pothos, Emmanuel M., \& Busemeyer, Jerome R. “A Quantum Probability Explanation for Violations of 'Rational’ Decision Theory.” Proc. Biol. Sci. 2009 June 22; 276 (1665): 2171-8. doi:10.1098/rspb.2009.0121

Rozema, Lee A., Darabi, Ardavan, Mahler, Dylan H., Hayat, A., Soudagar, Yasaman, \& Steinberg, Aephraim M. "Violation of Heisenberg's Measurement-Disturbance Relationship by Weak Measurements.” Phys, Rev. Lett. 109 (2012): $189-902$. 28/6/2018. arXiv:1208.0034 [quant-ph]

Rozema, Lee A., Mahler, Dylan H., Hayat, Alex, \& Steinberg, Aephraim M. “A Note on Different Definitions of Momentum Disturbance.” Quantum Studies: Mathematics and Foundations. 2013. 28/6/2018. arXiv:1307.3604v1 [quant-ph]

Schwartz, Jeffrey M., Stapp, Henry P., \& Beauregard, Mario. "Quantum Physics in Neuroscience and Psychology: A Neurophysical Model of Mind-Brain Interaction.” Phil. Trans. R. Soc. B 360 (2005): 1309-27. doi:10.1098/rstb.2004.1598

Schrödinger, Erwin. “An Undulatory Theory of the Mechanics of Atoms and Molecules.” Phys. Rev. 28.6 (1926): 1049-70.

Sorli, Amrit. “The Epistemological Crisis in Modern Physics.” Neuro Quantology 16.2 (2018): 1-5.

Stapp, Henry P. “Quantum Theory and the Role of Mind in Nature.” Foundations of Physics 31 (2001): 1465-99. $28 / 6 / 2018$. $<$ http://arxiv.org/abs/quant-ph/0103043>.

Straulino, S. “Reconstruction of Galileo Galilei’s Experiment: The Inclined Plane.” Physics Education 43.3 (2008): 316-21.

Tegmark, Max. Our Mathematical Universe: My Quest for the Ultimate Nature of Reality. New York, NY: Knopf A, 2014.

Von Neumann, John. Mathematical Foundations of Quantum Theory. Princeton, NJ: Princeton University Press, 1955.

Wikipedia (n.d.). Cloud Chamber. 12/10/2015. <https://en.wikipedia.org/wiki/Cloud_chamber>.

Zeh, Heinz D. “On the Interpretation of Measurements in Quantum Theory.” Found. Phys. 1 (1970): 69-76.

---. “The Role of the Observer in the Everett Interpretation.” Neuro Quantology 11.1 (2013): 97-105.

Zurek, Wojciech H. “Pointer Basis of Quantum Apparatus: Into What Mixture Does the Wave Packet Collapse?” Phys. Rev. D 24 (1981): 1516-25. 